\title{
Deep Learning-Based Phenotyping of Breast Cancer Cells Using Lens-free Digital In-line Holography
}

\author{
Tzu Hsi Song ${ }^{1,2}$, Mengzhi Cao ${ }^{3}$, Jouha Min ${ }^{4,5}$, Hyungsoon $\mathrm{Im}^{4}$, Hakho Lee ${ }^{4}$, Kwonmoo Lee ${ }^{1,2}$ \\ ${ }^{1}$ Department of Biomedical Engineering, Worcester Polytechnic Institute, MA, USA \\ ${ }^{2}$ Vascular Biology Program and Department of Surgery, Boston Children's Hospital, Harvard. Medical \\ School, Boston, MA, USA \\ ${ }^{3}$ Data Science Program, Worcester Polytechnic Institute, Worcester, MA, USA \\ ${ }^{4}$ Center for Systems Biology, Massachusetts General Hospital, Harvard Medical School, Boston, MA, \\ USA \\ ${ }^{5}$ Present Address: Department of Chemical Engineering, University of Michigan Ann Arbor, MI, USA \\ Corresponding Authors: Kwonmoo Lee and Hakho Lee
}

\begin{abstract}
Lens-free digital in-line holography (LDIH) produces cellular diffraction patterns (holograms) with a large field of view that lens-based microscopes cannot offer. It is a promising diagnostic tool allowing comprehensive cellular analysis with high-throughput capability. Holograms are, however, far more complicated to discern by the human eye, and conventional computational algorithms to reconstruct images from hologram limit the throughput of hologram analysis. To efficiently and directly analyze holographic images from LDIH, we developed a novel deep learning architecture called a holographical deep learning network (HoloNet) for cellular phenotyping. The HoloNet uses holo-branches that extract large features from diffraction patterns and integrates them with small features from convolutional layers. Compared with other state-ofthe-art deep learning methods, HoloNet achieved better performance for the classification and regression of the raw holograms of the breast cancer cells stained with well-known breast cancer markers, ER/PR and HER2. Moreover, we developed the HoloNet dual embedding model to extract high-level diffraction features related to breast cancer cell types and their marker intensities of ER/PR and HER2 to identify previously unknown subclusters of breast cancer cells. This
\end{abstract}


hologram embedding allowed us to identify rare and subtle subclusters of the phenotypes overlapped by multiple breast cancer cell types. We demonstrate that our HoloNet efficiently enables LDIH to perform a more detailed analysis of heterogeneity of cell phenotypes for precise breast cancer diagnosis.

\section{Introduction}

Breast cancer exhibits significant inter-tumor and intra-tumor heterogeneity, presenting significant diagnostic and therapeutic. Therefore, breast cancer tissues are biopsied to determine the hormone and growth factor receptor status for effective treatment because the hormonal status significantly affects the progress and phenotypes of breast cancer [1-3]. Specifically, the levels of nuclear estrogen (ER) or progesterone receptors $(\mathrm{PR})$ are measured to determine whether breast cancer cells will respond to anti-estrogens therapy using tamoxifen, fulvestrant or aromatase inhibitors. Also, human epidermal growth factor receptor 2 (HER2) is a tyrosine kinase receptor on the surface of breast cancer cells, and HER2-positive cancers are much more likely to benefit from anti-HER2 treatment with Herceptin $[1,3]$. Based on this hormone status, breast cancer can be identified into four different types: ER/PR-HER2-, ER/PR-HER2+, ER/PR+HER2+, ER/PR+HER2-. Moreover, several breast cancer subtypes have been identified in recent years, based on molecular and gene expression, and related clinical treatments are being developed [2]. Precise diagnosis and analysis of these breast cancer types, or subtypes out of heterogeneous tissue samples can provide more efficient and better treatments on breast cancer patients. However, such diagnosis is severely hampered by the limited data throughput and high cost of the current diagnosis workflow based on light microscopes. 
Lens-free digital in-line holography (LDIH) has been developed to address this challenge. LDIH is a powerful imaging technique that extracts the $3 \mathrm{D}$ positional information of an object into a single shot of 2D interference patterns, i.e., hologram images, and computationally reconstructs a three-dimensional (3D) image of the object. In addition, LDIH has a deep observation depth that can overcome the technical limitations and extract more detailed information than conventional microscopes. LDIH has been used in various fields, such as biological sample monitoring [4-6] and cell dynamic analysis [7-8], because holographic diffraction can be obtained to extract 3D cell morphological and biochemical information in a wide field of view [3]. But there are some issues with reconstructing 3D image from a hologram image. However, reconstructing 3D images requires substantial computational resources and time, and the artifact or information loss could happen. Alternatively, if we directly use raw holograms, it is much challenging to decern meaningful features by human vision and cognition, although the diffraction patterns contain rich information.

Deep learning (DL) has recently revolutionized machine learning and is highly capable of analyzing complex, large, and high-dimensional datasets [9-11]. DL approaches can learn meaningful features within complex datasets to analyze uncharacterized image patterns and recognize hidden patterns [9-11]. Due to these advantages of feature learning, DL approaches can effectively deal with the diffraction images because the features of the diffraction patterns cannot be directly recognized or analyzed by human intuition. Moreover, DL needs a large amount of data for effective model training. Because LDIH can capture large-scale datasets with a large field of view, LDIH can help DL algorithms avoid overfitting issues and build more robust neural network models. Also, since DL approaches directly learn complex diffraction patterns, they can avoid the errors or artifacts during reconstruction processing and make the LDIH applications robust. 
In recent years, DL approaches have been applied to LDIH, including reconstruction improvement $[12,13]$, phase retrieval [14], and classification and monitoring of various biological samples [3, 15, 16]. Min et al. developed an artificial intelligence diffraction analysis (AIDA) platform to make automated, rapid, high-throughput, and accurate cancer cell analysis [3]. AIDA platform allowed for quantitative molecular profiling of holograms from individual cells and revealed cellular heterogeneity. This platform can also directly perform cell recognition and color classification from raw holograms by Convolutional Neural Network (CNN) models. Moreover, Kim et al. have developed a deep transfer learning (DTL) approach to directly classify raw holograms generated from cells and microbeads without a reconstruction process [17]. DTL model extracts feature information using the pretrained VGG19 model as a general-purpose feature extractor [17] to identify and count microbeads on cells.

In this paper, we designed and implemented a novel deep learning approach analyze holograms from LDIH for breast cancer cell classification and analysis. Figure 1 shows the overview of our computational framework. Firstly, we used ER/PR and HER2 immuno-stained holograms as input data. Then we developed a novel holographic deep learning architecture, termed to HoloNet learn cellular diffraction features efficiently. We used this model to identify breast cancer cell types and predict ER/PR and HER2 intensity values. Here, we demonstrated that the proposed HoloNet could efficiently extract cellular hologram features to precisely classify different cell types and estimate intensity values. After cell classification and intensity regression, a holographical deep learning network with a dual embedding model is built to learn holographic feature vectors to generate feature distribution maps. These feature distribution maps are processed by manifold learning and unsupervised clustering methods to obtain previously unknown subclusters in breast cancer cell types. 


\section{Results}

\section{Overview of Proposed Workflow}

As described in Figure 1, the holograms were acquired by an LDIH imaging system form the breast cancer cell line (MCF7, T47D, SKBR3, BT474, and MDA-MB-231) immune-stained with anti-ER/PR and anti-HER2 conjugated with chromogens. We designed a novel holographic deep learning model to classify four breast cancer cell types: ER/PR-HER2-, ER/PR-HER2+, ER/PR+HER2+, and ER/PR+HER2- and predict intensity values of ER/PR and HER2 immunostaining. Second, we advanced our holographical deep learning model to extract high-level features to generate feature distribution maps. Using these features, we identified previously unknown subclusters hidden in heterogeneous samples by combining manifold learning and unsupervised clustering.

\section{Hologram Classification by HoloNet}

We design a novel deep learning holographical network (HoloNet) to extract and analyze holographical features. Figure 2(a) shows the architecture of the proposed HoloNet model. A holo-block is built to combine local details of objects with global features using a large kernel size of the convolutional filter and a concatenated layer. The HoloNet architecture is combined with a softmax layer as the output layer to classify breast cancer cell types. The cell classification results of the proposed HoloNet model are shown in Figure 2(b). Here we compare the classification performance with two types of input images, holograms and reconstructed images. We also used CNN [18-20] and Resnet [21] models to compare with the HoloNet model. First, we found that holograms provide more efficient features than reconstructed images in breast cancer cell classification. Even though the reconstructed images are built from holograms, the detailed 
information may be lost during the reconstruction processing, as we mentioned before. In addition, the HoloNet model with holograms has better performance than other DL models with holograms or reconstructed images. The HoloNet model provides better accuracy and F1-score than different approaches and achieved 95.5\% classification performance (Figure 2(b)). Although our HoloNet model has better classification performance than Densenet [22], Densenet almost provides similar performance as the HoloNet model does. It is because the HoloNet model and Densenet both used a similar concept of combining multiple-scale image features. Also, the proposed HoloNet model has better accuracy than previous work [3] over 5\% increase for classifying breast cancer cell types.

\section{Hologram Regression by HoloNet}

The marker intensities of ER/PR or HER2 are the important features of phenotyping breast cancer cells. Conventionally, Therefore, we quantify their intensities directly from raw hologram using HoloNet with the fully connected layer of the regression output. The HoloNet regression model can directly and precisely obtain intensity values of ER/PR and HER2 channels without the reconstruction process. Figure 2(c) shows that the HoloNet model can efficiently provide the ability of intensity prediction in both ER/PR and HER2 staining channels, which the $R^{2}$ scores are 0.9743 and 0.9795 , respectively. Here we also use the network structures of CNN [18-20] and Resnet [21] to predict the intensity values and compare the outcomes with our HoloNet model. We found that the HoloNet model can predict the more accurate intensities of both staining channels than other DL models.

\section{Sub-clustering of Breast Cancer Cells with HoloNet Dual Embedding}

Since deep learning models can extract rich features from input images, we further develop hologram feature embedding methods to identify previously uncharacterized subtypes of breast 
cancer cells. Figure 3(a) shows that the workflow of feature extraction and sub-clustering analysis. We developed HoloNet dual embedding learning model, called Dual HoloNet, to obtain the diffraction feature vectors. The hologram features extracted from HoloNet are used for the cell type classification and the intensity regression simultaneously. This structure will help the HoloNet learn the features for subclustering in each breast cancer cell type while paying more attention to the features related to the intensities. Therefore, the resulting subclusters can have differential intensity distributions in each cell type. Then the feature vector is obtained from the Dual HoloNet model and processed by Uniform Manifold Approximation and Projection (UMAP) method [23] to learn the feature manifold and reduce the dimension of features. Figure 3(b) and 3(c) show the feature distributions of breast cancer cells obtained by the HoloNet with holographic input images and reconstructed input images, respectively. The feature distribution map from the reconstructed input images shows that the clusters of different cell types can be distinctly separated because of the distance. But, due to the lack of intra-cluster heterogeneity, it is difficult to find potential subclusters. The feature distribution map from holograms exhibited high levels of intra-cluster heterogeneity while the inter-cluster distances become smaller than the features from the reconstruction images. In contrast, the hologram distribution map from the Dual HoloNet model in Figure 3(d) showed the sizeable intra-cluster heterogeneity and inter-cluster distances, suggesting that the features from Dual HoloNet embedding are suitable for sub-clustering analysis.

To determine the optimal number of subclusters in each cell type, we combined community detection with spectral clustering [24] and grid search. We used clustering evaluation functions to estimate the cohesion values of subclusters and used rank voting to select the optimal number of subclusters in each class. To determine the optimal balance between the branches of classification and regression, we varied the ratio of two loss weights and evaluated the sub-clustering results by 
the mean intensity differences of ER/PR and HER2 among the subclusters. Figure 3(e) shows the mean intensity values in different ratios of the loss of classification and regression, and we chose the embedding that provided the maximum mean intensity differences.

\section{Subclustering Analysis in Cell Line Samples}

Figure 4(a) represents the subcluster distribution maps with the optimal loss weight in each cell type. We obtained four subclusters with loss weight ratio 1:1 in ER/PR-HER2-, four subclusters with loss weight ratio 5:1 in ER/PR-HER2+ and ER/PR+HER2- cell types, and three subclusters with loss weight ratio 5:1 in ER/PR+HER2+. After the subclusters were obtained in different cell type groups, we observed the cell population of subclusters in cell line sample cases. In Figure 4(b), we found that MCF7 and T47D cell lines are dominated by ER/PR+HER2- cell type, but they consist of markedly different distribution of the subclusters. While Cluster 13 is the major subcluster in MCF7, Cluster 15 is the major component in T47D. Moreover, the SKBR3 cell line whose major cell type is ER/PR+HER2+ consists of Cluster 9-11 equally. In the BT474 cell line, Cluster 6 and 7 dominate ER/PR-HER2+ cell type. MDA-MD-231 cell line where most of the cells belong to ER/PR-HER2- mainly composed Cluster 1-3. In addition, Cluster 3 and 4 exist in ER/PR-HER2- of the MCF7 cell line, and Cluster 8 mainly dominates the ER/PR-HER2+ cell type of the SKBR3 cell line.

To know the natures of these subclusters, we quantified their average intensities and the distributions of the breast cancer cell lines in each subcluster. Because our hologram embedding was designed to identify the features partially discriminative to the marker intensities, the mean intensity values of the subclusters were generally statistically different (Figure 5(a)-(d)). In ER/PR-HER2- cell type, the mean subcluster intensities in both channels were gradually increased from Cluster 1 to 4 (Figure 5(a)). Most of the cells in Cluster 1 and 2 whose mean intensities are 
low were from MDA-MB-231. Cluster 3 has a mixed population of MDA-MB-231 and MCF7. Cluster 4, whose mean intensities are the highest, mainly consists of MCF7 along with minor populations from T47D, BT474, and MDA-MB-231 (Figure 5(e) and (i)). In ER/PR-HER2+ cell type, the subclusters also had different mean intensities (Figure 5(b)). Cluster 5, 6, and 7 were mainly from BT474. In Cluster 8, whose intensities are the highest among the subclusters, BT474 and SKBR3 co-existed equally (Figure 5(f) and (j)). In ER/PR+HER2+ cell type, the mean intensities of ER/PR channel increased from Cluster 9 to 11 while the mean HER2 intensities decreased (Figure 5(c)). The major cell line in these subclusters is SKBR3, but Cluster 9 has a minor cell population from BT474 (Figure 5(g) and (k)). In ER/PR+HER2- cell type, the mean intensities in both channels increased from Cluster 12 to 15 (Figure 5(d)). Cluster 12 and 13 consisted of MCF7 along with minor proportions of T47D. Cluster 14 and 15 consisted of T47D along with minor proportions of MCF7 in Cluster 14 and SKBR3 in Cluster 15 (Figure 5(h) and (l)).

Table 1. Summary of the distributions of breast cancer cell lines in the identified subclusters.

\begin{tabular}{|c|c|c|c|c|c|c|c|c|c|c|c|c|c|c|c|}
\hline Cell Type & \multicolumn{3}{|c|}{ ER/PR-HER2- } & \multicolumn{3}{c|}{ ER/PR-HER2+ } & \multicolumn{3}{c|}{ ER/PR+HER2+ } & \multicolumn{3}{c|}{ ER/PR+HER2- } \\
\hline Subcluster & 1 & 2 & $\mathbf{3}$ & $\mathbf{4}$ & 5 & 6 & 7 & $\mathbf{8}$ & $\mathbf{9}$ & 10 & 11 & 12 & 13 & 14 & $\mathbf{1 5}$ \\
\hline MCF7 & & & + & ++ & & & & & & & & ++ & ++ & + & \\
\hline T47D & & & & + & & & & & & & & + & + & ++ & ++ \\
\hline SKBR3 & & & & & & & & + & ++ & ++ & ++ & & & & + \\
\hline BT474 & & & & + & ++ & ++ & ++ & + & + & & & & & & \\
\hline $\begin{array}{c}\text { MDA-MB- } \\
231\end{array}$ & ++ & ++ & ++ & + & & & & & & & & & & & \\
\hline
\end{tabular}

As summarized in Table 1, in Cluster 3, 4, 8, 9, and 15, the cells from different cell types co-exist. Cluster 3 is near the boundary between ER/PR-HER2- and ER/PR+HER2-. Cluster 4 is near the boundary among ER/PR-HER2-, ER/PR-HER2+ and ER/PR+HER2-. Cluster 8 and 9 are near the boundary between ER/PR-HER2+ and ER/PR+HER2-. Cluster 15 is near the boundary between 
ER/PR+HER2- and ER/PR+HER2+. While there exist breast cancer cells whose phenotypes are near characteristics near the boundaries among the previously known cell types, these cells were treated to belong to a single cell type for a diagnostic purpose previously. We used our hologram embedding to identify those subclusters of breast cancer cells sharing similar characteristics among the known cell types.

\section{Characterizing the Heterogeneity of Breast Cancer Cells from Patients}

The previous results are based on the multiple breast cancer cell lines. To confirm the clinical significance of these subclusters, we used two the hologram data from two breast cancer patient cases used in the previous study [3]. Here we used the proposed HoloNet model of classification to identify the cell type distribution of these patient cases. Figure 6(a) shows the proportions of the cell types from two breast cancer patients. While the cell types of the most significant proportion are ER/PR-HER2- in Patient case 1 and ER/PR+HER2- in Patient case 2, their overall cell type distributions are marginally different. We used our hologram embedding to obtain feature vectors of cellular holograms from these patients and profile them with our subclusters of breast cancer cells. In Figure 6(b), we found that Patient case 1 has much more Cluster 1 from ER/PRHER2- than Patient case 2 while Patient case 2 has much more Cluster 4 from ER/PR-HER2- than Patient case 1 . Given our finding that Cluster 4 share the phenotypes from ER/PR-HER2+ and ER/PR+HER2-. The characteristics of ER/PR-HER2- in these two patients are distinct.

For ER/PR+HER2- type, two patients have the similar largest proportions of Cluster 15, sharing the similar characteristics of ER/PR+HER2+. However, Patient case 1 has a much more proportion of Cluster 13 than Patient case 2, while Patient case 2 has much more Cluster 12. Since there are significant differences in both channels between Cluster 12 and 13, the cellular phenotypes in ER/PR+HER2- of these patients are also distinct. Taken together, we demonstrated that some of 
the identified subclusters existed in the breast cancer patient samples, and the subclustering results can provide much richer information on the disease status.

\section{Discussion}

We developed the HoloNet, which can efficiently learn high-level diffraction features from the complex holograms to precisely discriminate breast cancer cell types in both supervised and unsupervised learning setting. Especially, the holo-block unit adapts different large-scale filters to collect multi-scale feature information to identify detailed local cellular features. It is because the local feature information in holograms does not correspond to a particular part of cellular images but rather the entire images. These large-scale filters applied to holograms can collect more related local cellular information. Our HoloNet can efficiently extract cell information from holograms to provide better performances of cell classification and intensity regression than other existing deep learning models.

We demonstrated that the feature embedding directly from holograms enabled us to identify detailed subclusters of breast cancer cells. We added the intensity regression into the Dual HoloNet model along with the classification of the previously known cell types. This structure was able to help the neural network pay more attention to specific hologram features to enhance the difference of the marker intensities among different cell types. Then we optimized the loss weights to maximize the differences of the marker intensities among potential subclusters. This hologram embedding allowed us to identify the subclusters within the known cell types for refined cellular phenotyping. Some of the subclusters identified in our study have the phenotypes shared by multiple breast cancer cell types since they are located near the class boundaries in the feature 
space. Identifying these rare and subtle cellular phenotypes can be significant in clinical decisionmaking because they may have different drug sensitivity and resistance from the previously known cell types. We expect that HoloNet, in conjunction with LDIH, opens a new opportunity to fully characterize intra/inter-tumor heterogeneity in breast cancer and provide clinicians with valuable information for patient-specific breast cancer therapy.

\section{Methods}

\section{Data collection}

Breast cancer cells were captured by the surface coated by antibodies (HER2, EpCAM, EGFR, and MUC1 [3]) and bio-adhesives, and then stained by anti-ER/PR and anti-HER2 conjugated with chromogen. Then cellular holograms were obtained by a LDIH system, and the image patches containing a cell were cropped with 64 X64 pixel size in both hormonal staining channels. These image data include four different cell types: ER/PR-HER2-, ER/PR-HER2+, ER/PR+HER2-, and ER/PR-HER2+, from five cell lines (MCF7, T47D, SKBR3, BT474, and MDA-MB-231) [3]. The number of all holograms was 5026. For more efficient training, data augmentation was applied to balance the size of different cell types by using random rotation and flipping. Then we separated the data into training (65\%), validation (15\%), and testing (20\%) with 5-fold cross validation for model evaluation. The ground truth of intensity values of ER/PR and HER2 of cell images were obtained from the reconstructed images.

\section{Holographical deep learning network (HoloNet)}

We constructed the HoloNet based on the concept of convolutional neural network [18-20] by adding several large-kernel-size filters to efficiently extract hologram features. Here we designed 
a block called holo-block, which combines local and global holographical features. The parameters

$\mathrm{K}, \mathrm{L}$, and $\mathrm{s}$ in a holo-block (Figure 2(a)) represent the kernel size of the convolutional layer, number of a feature layer, and sliding, respectively. There are three holo-blocks with different kernel size of convolutional layers, which are 16X16, 24X24, and 32X32, respectively, and average pooling layers are used to obtain and emphasize specific feature information. The number of feature layer in these blocks was 64 . The sliding numbers were set to 1,2 , and 4 for each holoblock. Moreover, a convolutional layer with batch normalization and three fully connected layers are connected to these holo-blocks to build the HoloNet model. Rectified linear unit (ReLu) was used as an activation function in the model [25]. Based on the HoloNet architecture, we implemented a DL model to classify four types of breast cancer cells by a softmax layer. Then, for the intensity regression, we constructed the HoloNet architecture with a fully connected layer to predict intensity values of ER/PR and HER2 staining channels from holograms.

\section{HoloNet dual embedding model (Dual HoloNet)}

The architecture of HoloNet dual embedding model (Dual HoloNet) includes the HoloNet model with two fully connected layers of intensity regression and the cell type classification (Figure 3(a)). The total loss function of the training is shown as:

$$
\text { Loss }_{\text {Total }}=\alpha \times \text { LosS }_{\text {Classification }}+\beta \times \text { LosS }_{\text {Regression }}
$$

,where $\alpha$ and $\beta$ are the loss weights of classification and intensity regression for loss balancing. Here we used Brier loss [26] for the classification loss as below:

$$
\text { Loss }_{\text {classification }}=\frac{1}{N} \sum_{t=1}^{N} \sum_{i=1}^{R}\left(f_{t i}-o_{t i}\right)^{2}
$$


,where $\mathrm{N}$ is the number of observations and $\mathrm{R}$ means the number of categorical labels. $f$ and $o$ represent the predictive and true label distribution, respectively. Brier loss function is similar to mean square error loss but has the same ability of loss energy as the cross-entropy function.

\section{Neural Network Training}

We used Adam optimizer with learning rate $=10^{-4}$ and batch size $=128$. The categorical crossentropy was used as a loss function for training the HoloNet model for cell classification, and the mean square error loss function was used for HoloNet model training for the intensity regression. We set the input image size as $64 \times 64 \times 2$ for the classification and $64 \times 64$ in each staining channel for the regression. For the HoloNet model, the maximum epoch was 100 for the classification and 500 for the regression. The pixel value of input image was normalized from 0 to 1 . We also automatically reduce the learning rate by multiplying with 0.1 in every 20 epochs. For training the dual HoloNet model, we set that brier loss function as a loss function for the classification and the maximum epoch $=150$. We used default parameters in the Keras library, and the environment in Python was TensorFlow 1.15 with CUDA 10.0 for both HoloNet models.

\section{Unsupervised Clustering and Subcluster Selection}

We used the second last layer of the Dual HoloNet model to extract the feature vector of 500 dimensions, and the UMAP method [23] was used to reduce these 500 dimensional feature vectors to three-dimensional space. In the parameters of the UMAP method, the number of the neighborhood was set to 20 and the dimension of the space was 3 . Also, the minimum distance among the observation was set to 0.1 , and the string metric was the correlation to compute distance in high dimensional space. Then, the pairwise distances between hologram feature vectors were calculated to generate a similarity matrix. The threshold value of similarity was set to 0.5 to build an adjacent matrix. This adjacent matrix was used as community detection to obtain subclusters 
by using spectral clustering [24]. The grid search method was used to find the optimal numbers of subclusters in each cell type. We set the number of subclusters from 1 to 10 and evaluated the clustering quality of different clustering numbers using clustering evaluation functions including silhouette coefficient [27], Dunn's index [28], Calinski-Harabasz index [29], and Davies-Bouldin index [30]. Then the optimal number of subclusters in each cell type was selected by voting the highest rank from the list of clustering values among different subclustering numbers.

\section{Loss Weight Optimization}

We used grid search to determine the optimal ratio of loss weight in each cell type for subclustering. We calculated Euclidean distances of the mean intensity values of ER/PR and HER2 channels among subclusters and then averaged those Euclidean distances. We evaluated this mean Euclidean intensity distance among the subclusters with the varying loss ratios: $1: 1,3: 1,5: 1,10: 1$ and 100:1 (classification : regression). Then we select the optimal weight combinations which provide the maximum intensity difference in each cell type (Figure 3(e)).

\section{Data availability statement}

The datasets used in the current study are available from the corresponding author on a reasonable request.

\section{Code availability statement}

The datasets used in the current study are available from the corresponding author on a reasonable request.

\section{Acknowledgement}


We thank Boston Scientific for providing us with the gift for deep learning research. This work was supported by DoD grants, W81XWH1910200 (K.L.) and W81XWH1910199 (H.L.).

\section{Competing Interests}

The author declare no competing financial or non-financial interests.

\section{Author Information}

Correspondence and requests for materials, data, and code should be addressed to K.L.

(kwonmoo.lee@childrens.harvard.edu) or H.L. (hlee@mgh.harvard.edu)

\section{Reference}

1. Nounou, Mohamed I., et al. "Breast cancer: conventional diagnosis and treatment modalities and recent patents and technologies." Breast cancer: basic and clinical research 9 (2015): BCBCRS29420

2. Goldhirsch A, et al. Personalizing the treatment of women with early breast cancer: highlights of the St Gallen International Expert Consensus on the Primary Therapy of Early Breast Cancer 2013. Ann Oncol. 2013;24(9):2206-23.

3. Min, Jouha, et al. "Computational optics enables breast cancer profiling in point-of-care settings." ACS nano 12.9 (2018): 9081-9090.

4. Mirsky, Simcha K., et al. "Automated analysis of individual sperm cells using stain-free interferometric phase microscopy and machine learning." Cytometry Part A 91.9 (2017): 893900 .

5. Singh, Dhananjay Kumar, et al. "Label-free, high-throughput holographic screening and enumeration of tumor cells in blood." Lab on a Chip 17.17 (2017): 2920-2932.

6. Yi, Faliu, Inkyu Moon, and Bahram Javidi. "Cell morphology-based classification of red blood cells using holographic imaging informatics." Biomedical optics express 7.6 (2016): 2385-2399.

7. Katz, Joseph, and Jian Sheng. "Applications of holography in fluid mechanics and particle dynamics." Annual Review of Fluid Mechanics 42 (2010): 531-555

8. Memmolo, Pasquale, et al. "Recent advances in holographic 3D particle tracking." Advances in Optics and Photonics 7.4 (2015): 713-755

9. LeCun, Y., Bengio, Y. \& Hinton, G. Deep learning. Nature 521, 436-444, https://doi.org/10.1038/nature14539 (2015). 
10. Schmidhuber, Jürgen. "Deep learning in neural networks: An overview." Neural networks 61 (2015): 85-117.

11. Rawat, Waseem, and Zenghui Wang. "Deep convolutional neural networks for image classification: A comprehensive review." Neural computation 29.9 (2017): 2352-2449.

12. Go, T., Lee, S., You, D. et al. Deep learning-based hologram generation using a white light source. Sci Rep 10, 8977 (2020). https://doi.org/10.1038/s41598-020-65716-4

13. Alexander, Ronald, Brian Leahy, and Vinothan N. Manoharan. "Precise measurements in digital holographic microscopy by modeling the optical train." Journal of Applied Physics 128.6 (2020): 060902.

14. Wu, Y. et al. Extended depth-of-field in holographic imaging using deep-learning-based autofocusing and phase recovery. Optica 5, 704-710 (2018).

15. Ren, Z., Xu, Z. \& Lam, E. Y. End-to-end deep learning framework for digital holographic reconstruction. Adv. Photonics 1, 016004 (2019).

16. Zhang, G. et al. Fast phase retrieval in off-axis digital holographic microscopy through deep learning. Opt. Express 26, 19388-19405 (2018).

17. Kim S-J, Wang C, Zhao B, Im H, Min J, Choi NR, Castro CM, Weissleder R, Lee H, Lee K (2018) Deep transfer learning-based hologram classification for molecular diagnostics. bioRxiv DOI:10.1101/192559

18. Krizhevsky, A., Sutskever, I. \& Hinton, G. E. Imagenet classification with deep convolutional neural networks. Advances in Neural Information Processing Systems. 1097-1105 (2012).

19. Zeiler, M. D. \& Fergus, R. Visualizing and understanding convolutional networks. European Conference on Computer Vision. 818-833 (Springer) (2014).

20. Oquab, M., Bottou, L., Laptev, I. \& Sivic, J. Learning and transferring mid-level image representations using convolutional neural networks. Computer Vision and Pattern Recognition (CVPR), 2014 IEEE Conference on. 1717-1724 (IEEE) (2014).

21. He, Kaiming, et al. "Deep residual learning for image recognition." Proceedings of the IEEE conference on computer vision and pattern recognition. 2016.

22. Huang, Gao, et al. "Densely connected convolutional networks." Proceedings of the IEEE conference on computer vision and pattern recognition. 2017.

23. McInnes, Leland, John Healy, and James Melville. "Umap: Uniform manifold approximation and projection for dimension reduction." arXiv preprint arXiv:1802.03426 (2018).

24. Van Gennip, Yves, et al. "Community detection using spectral clustering on sparse geosocial data." SIAM Journal on Applied Mathematics 73.1 (2013): 67-83

25. Hara, Kazuyuki, Daisuke Saito, and Hayaru Shouno. "Analysis of function of rectified linear unit used in deep learning." 2015 international joint conference on neural networks (IJCNN). IEEE, 2015

26. Brier, Glenn W. "Verification of forecasts expressed in terms of probability." Monthly weather review 78.1 (1950): 1-3 
27. Rousseeuw, Peter J. "Silhouettes: a graphical aid to the interpretation and validation of cluster analysis." Journal of computational and applied mathematics 20 (1987): 53-65

28. Dunn, Joseph C. "A fuzzy relative of the ISODATA process and its use in detecting compact well-separated clusters." (1973): 32-57.

29. Caliński, Tadeusz, and Jerzy Harabasz. "A dendrite method for cluster analysis." Communications in Statistics-theory and Methods 3.1 (1974): 1-27.

30. Davies, David L., and Donald W. Bouldin. "A cluster separation measure." IEEE transactions on pattern analysis and machine intelligence 2 (1979): 224-227. 


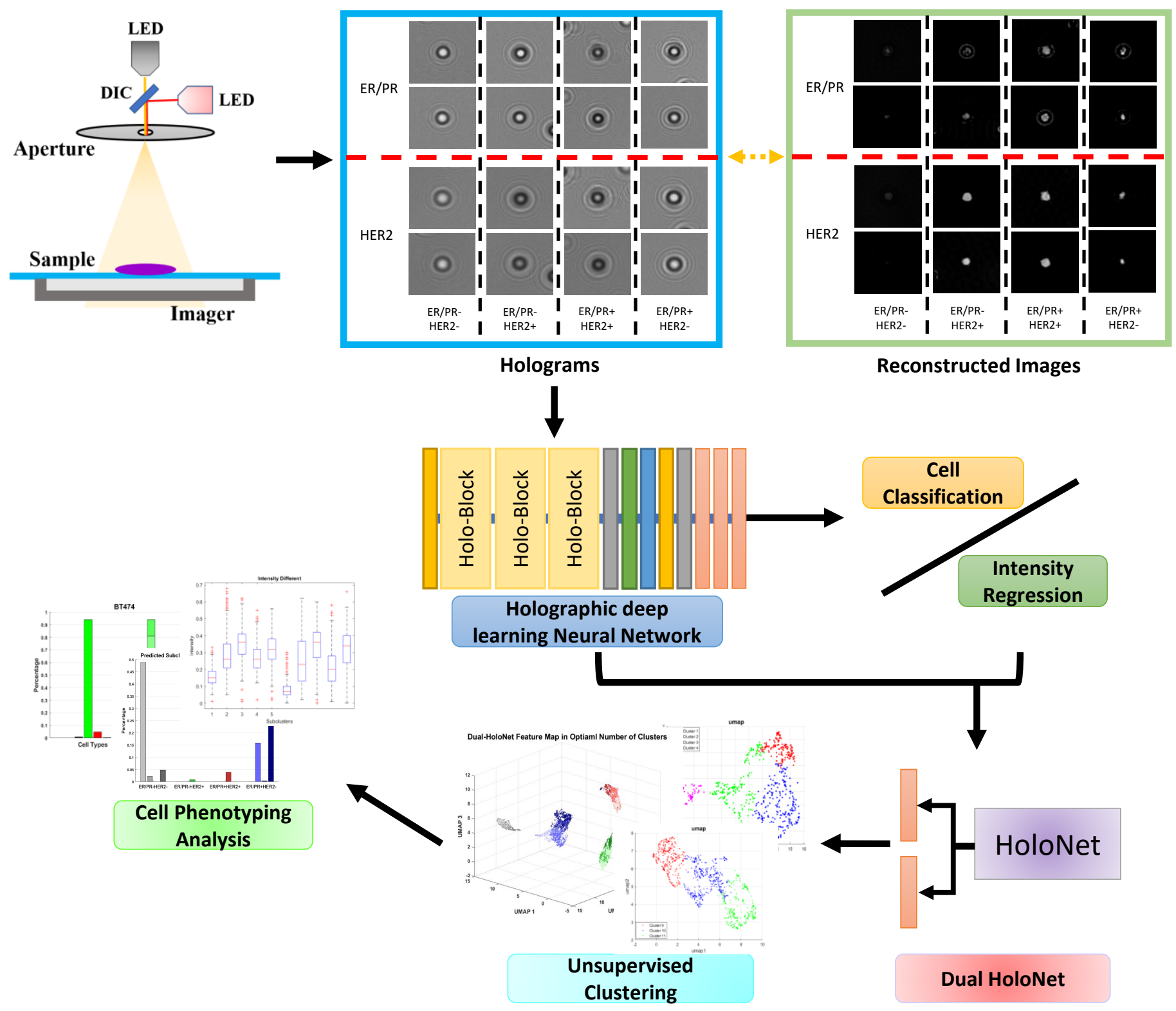

Figure 1. Workflow of the proposed deep learning models and the analysis of cell phenotyping in digital lensless inline holography. 
(a)

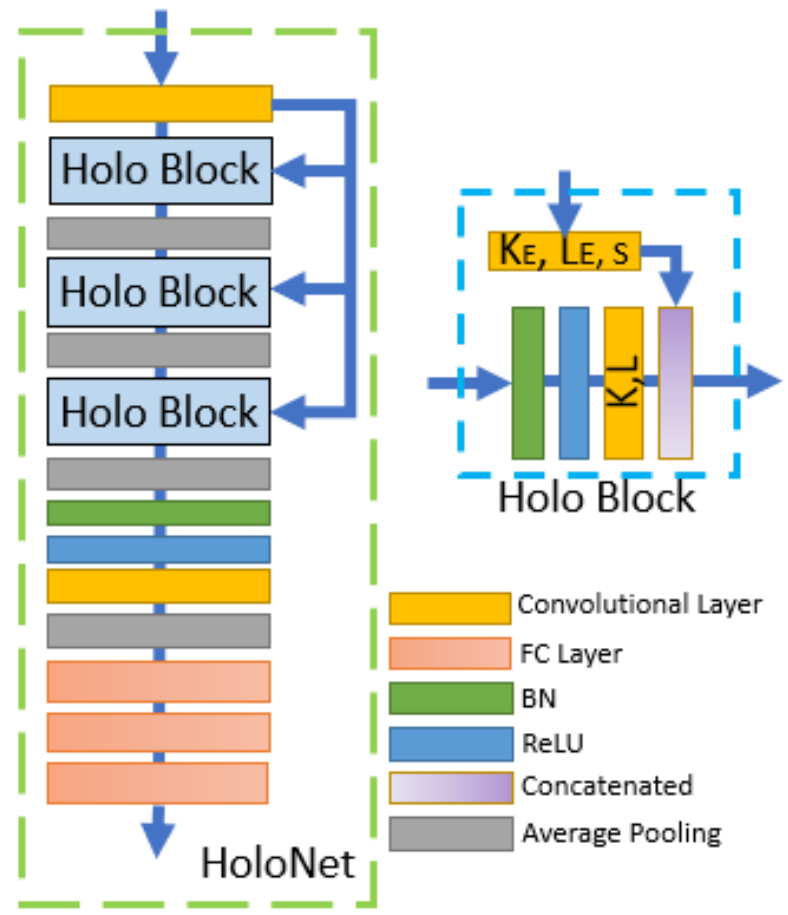

(c)
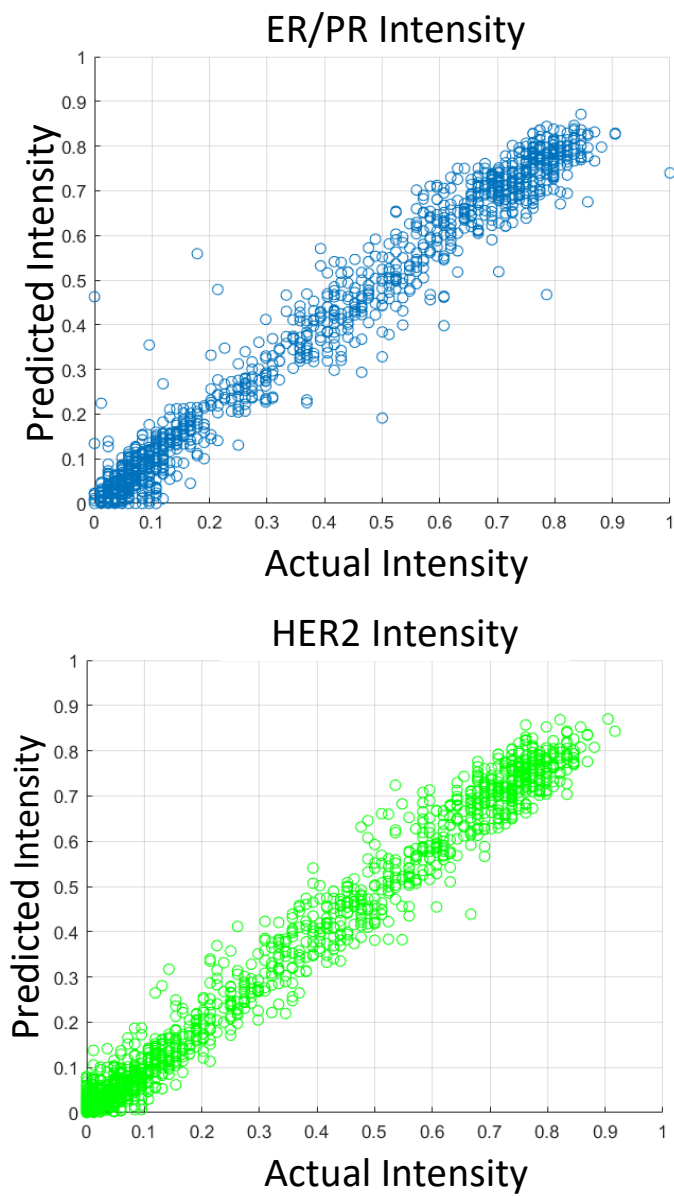

(b)

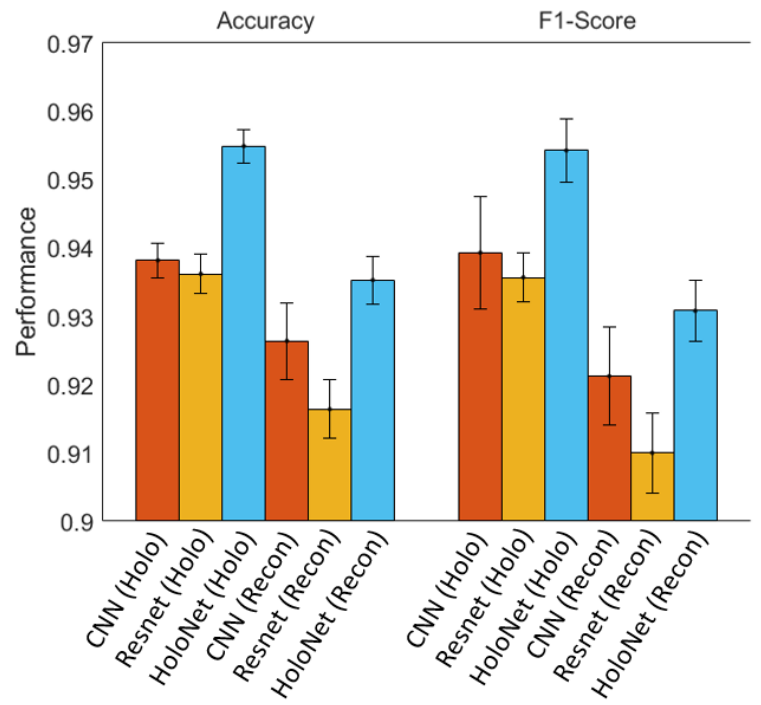

Cell Classification of Different Nework Models with Hologram Images

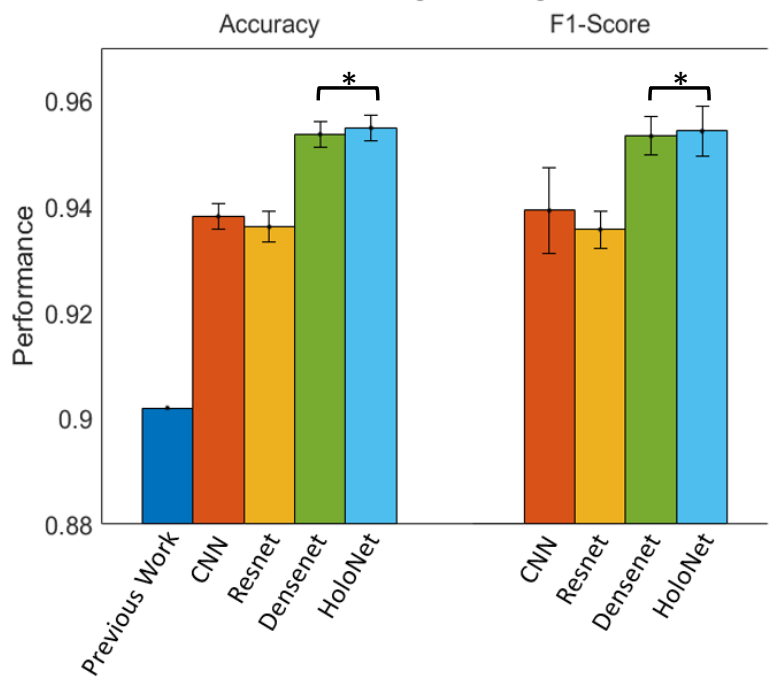

(d)

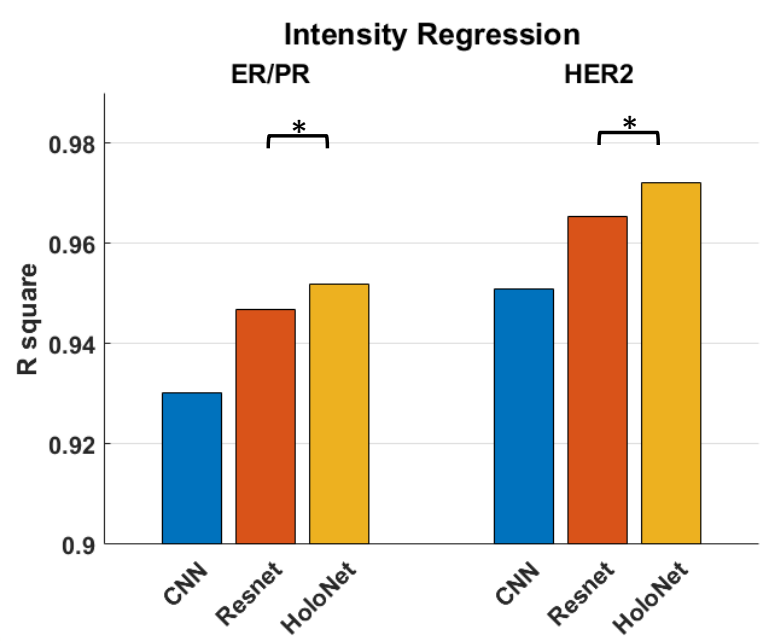

Figure 2. Structure and performance of the holographic deep learning network (HoloNet). (a) The architecture of the HoloNet. (b) Comparison of the performances of cell type classification between holograms and reconstructed images with different deep learning structures. (c) Intensity regression results from the HoloNet. (d) Comparison of the regression performance between different deep learning structures. * indicates the statistical significance $(\mathrm{p}<0.01)$ 
(a)
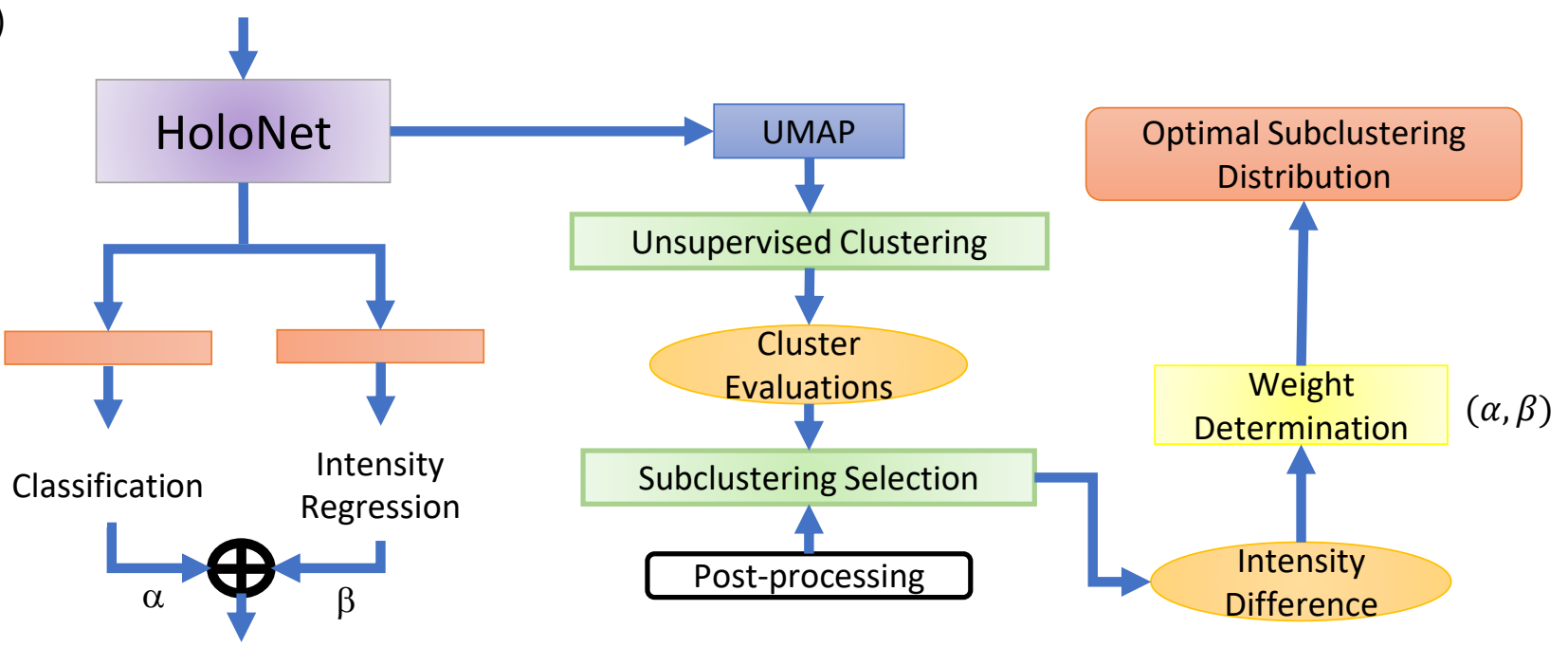

Combined Loss

(b)

HoloNet with holographic images

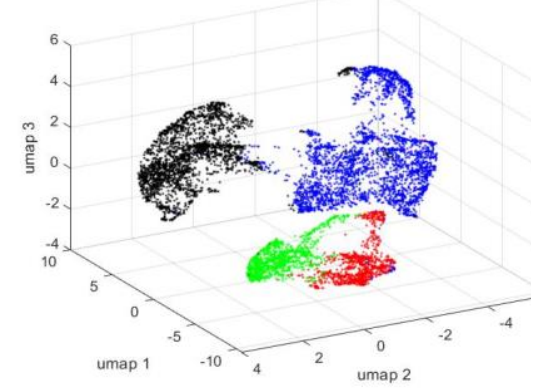

(d)

Dual HoloNet with holographic images

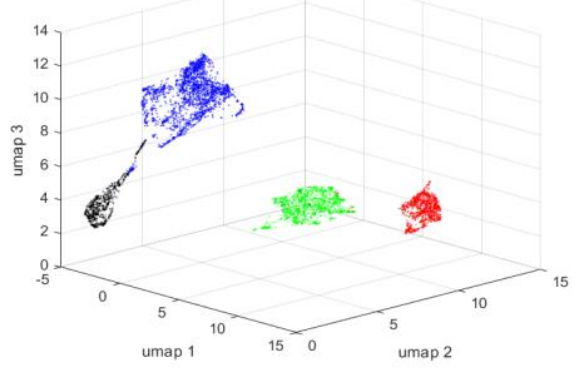

(c)

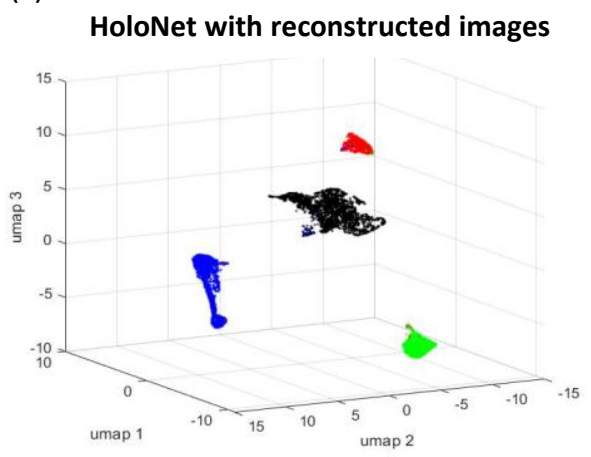

- ER/PR-HER2-

ER/PR-HER2+

- ER/PR+HER2+

- ER/PR+HER2-

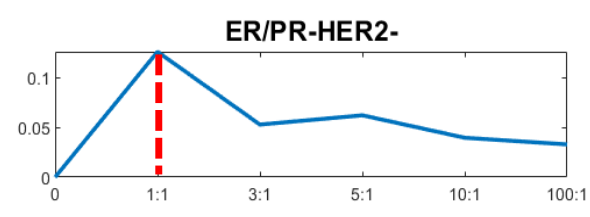

ER/PR-HER2+

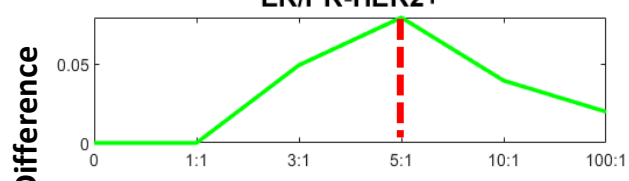

ER/PR+HER2+

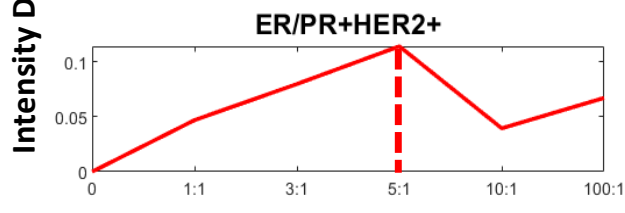

ER/PR+HER2-

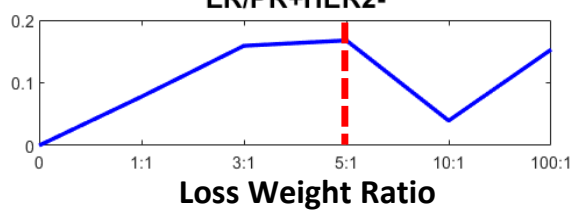

Figure 3. Pipeline of HoloNet-based unsupervised learning. (a) The architecture of the HoloNet dual embedding model (Dual HoloNet) with unsupervised subclustering selection. (b-d) Feature distribution maps from the HoloNet with holograms (b), the HoloNet with reconstructed images (c). the Dual HoloNet with holograms (d). (e) Determination of the loss weight in each cell type. 
(a)
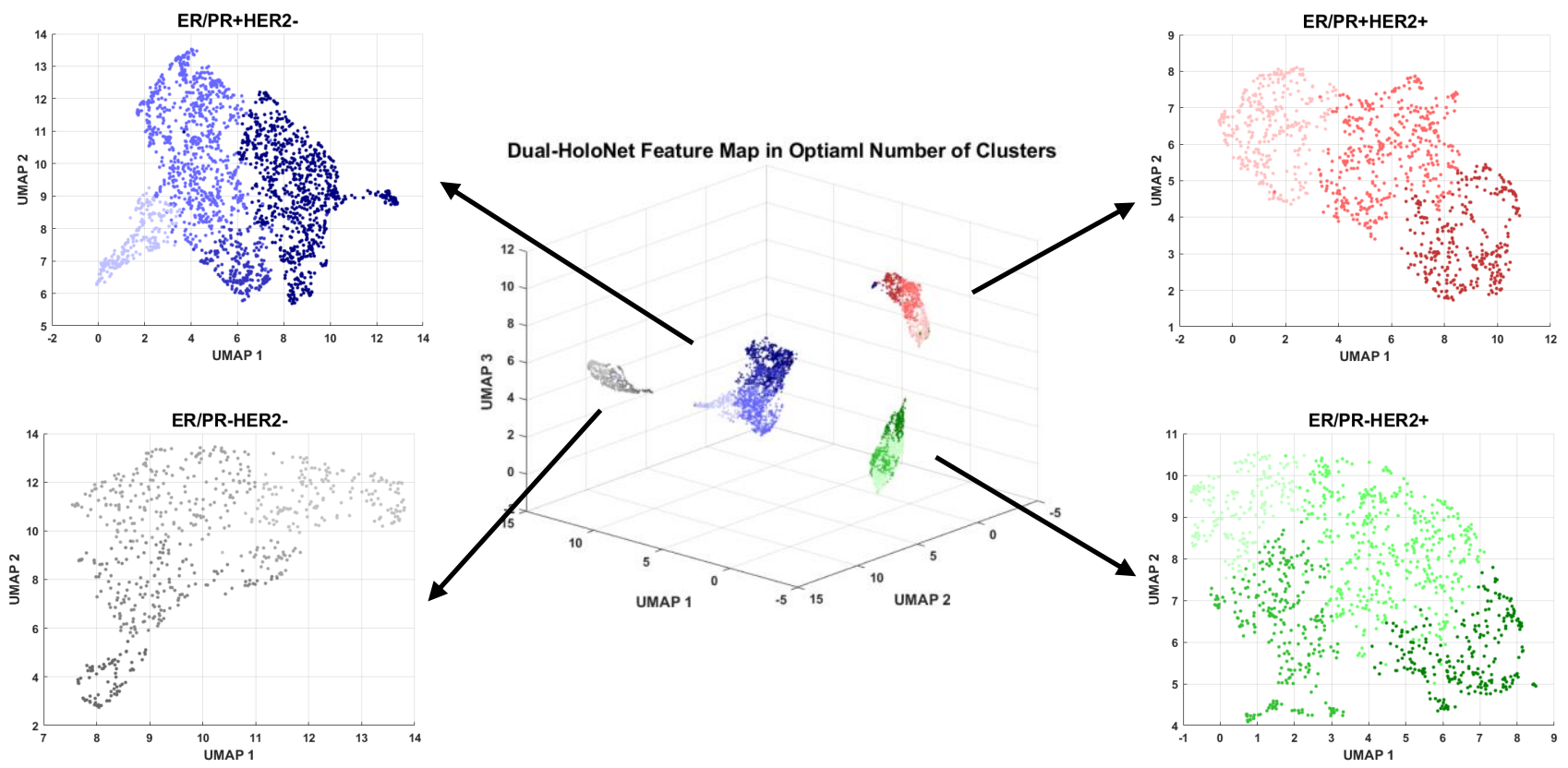

(b)

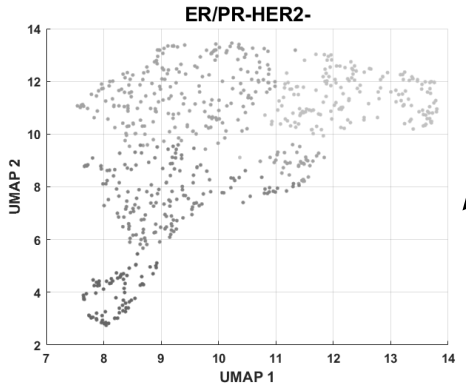

Cell Line Samples with Subclustering

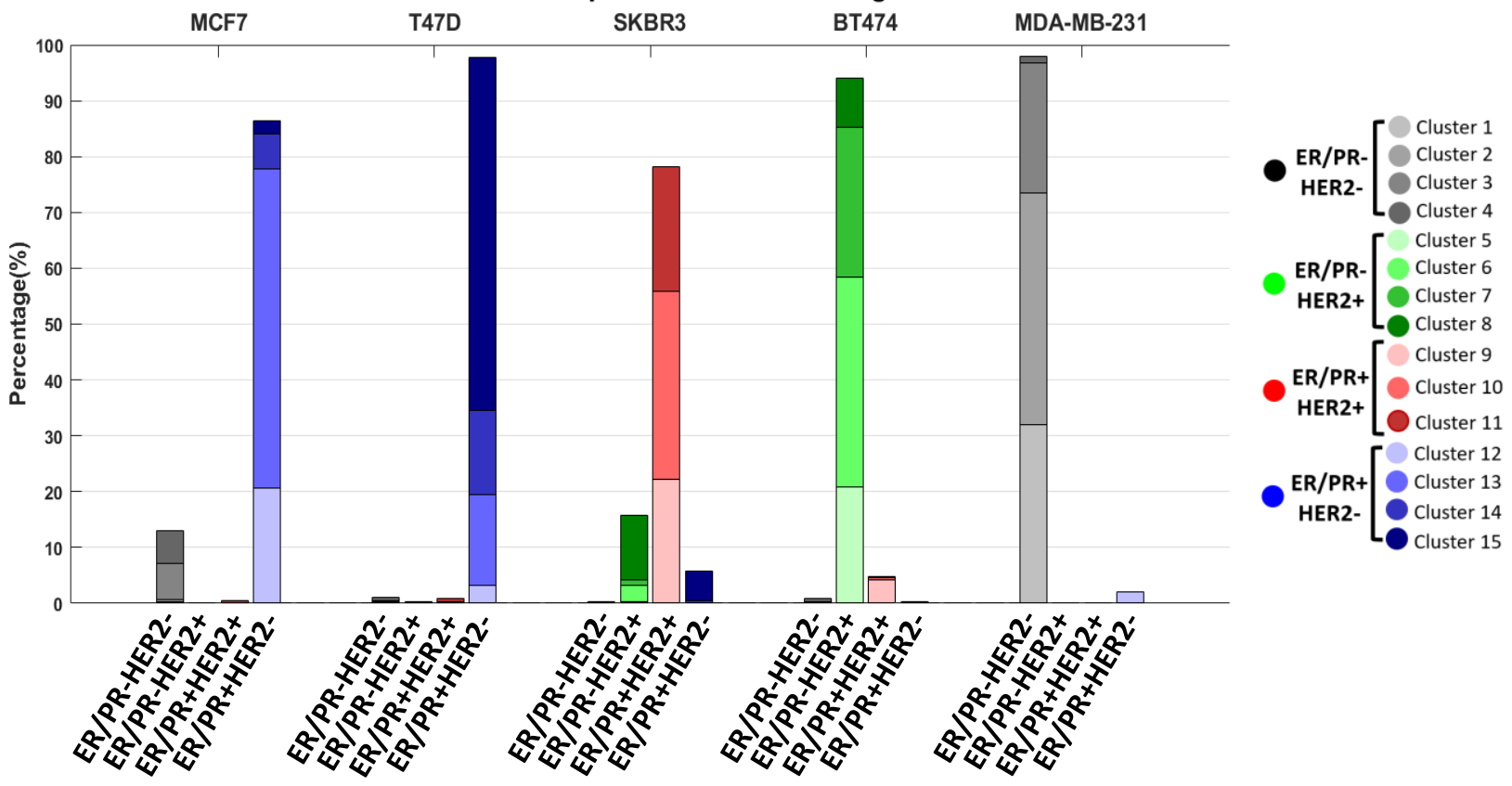

Figure 4. Subcluster results from hologram embedding. (a) Distribution of hologram features from Dual HoloNet embedding and the subclustering results in each cell type. (b) Subcluster distribution in each cell line. 
(a)

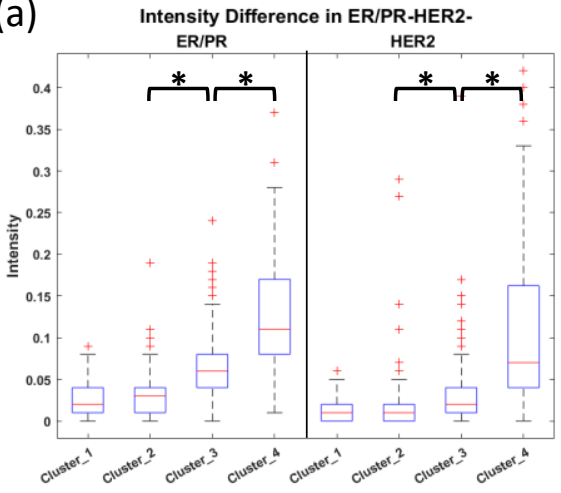

(b)

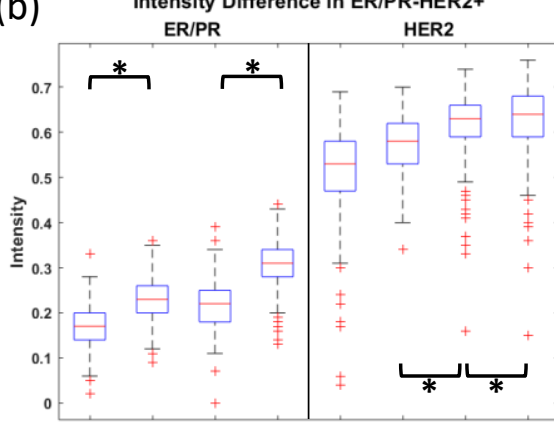

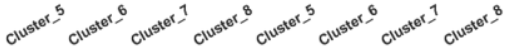

(c)

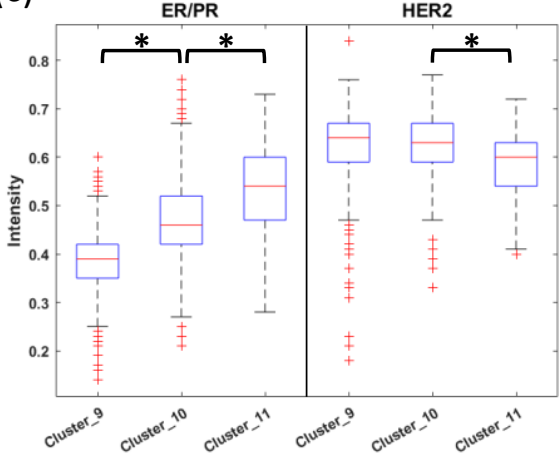

(d)

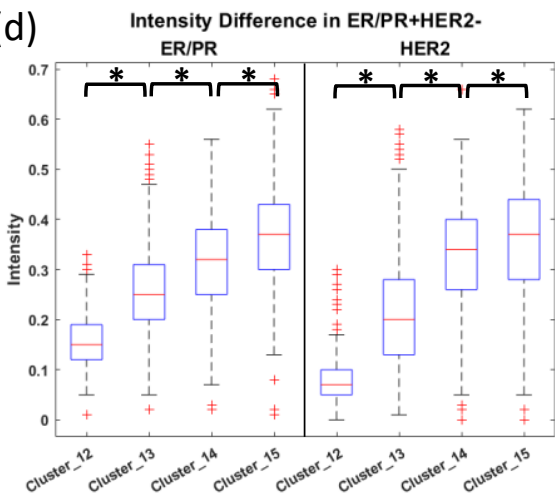

(e)

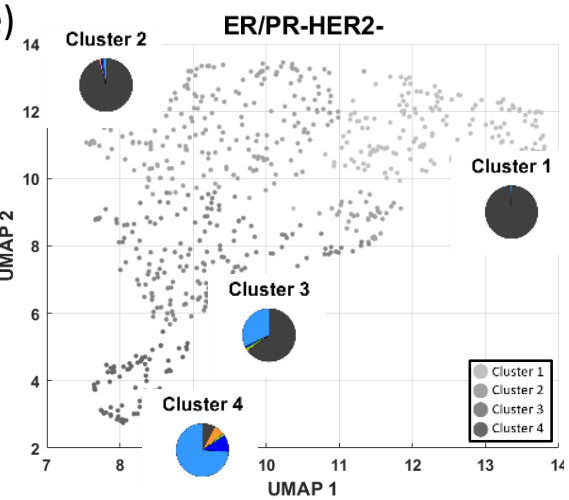

(f)

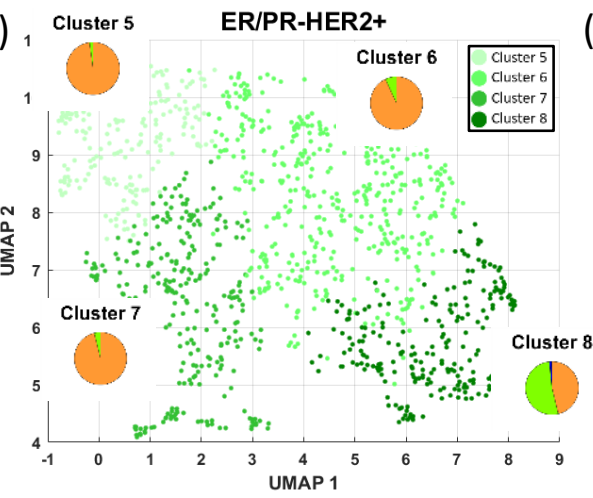

(g)

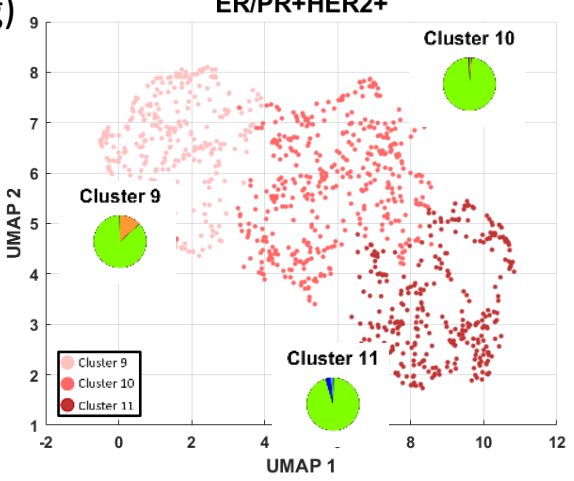

(h)

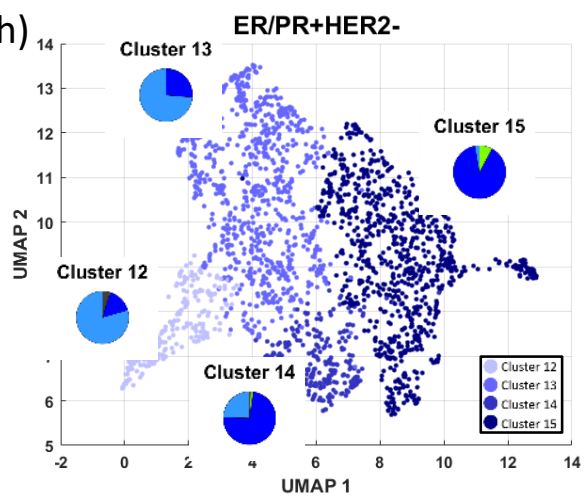

(i)
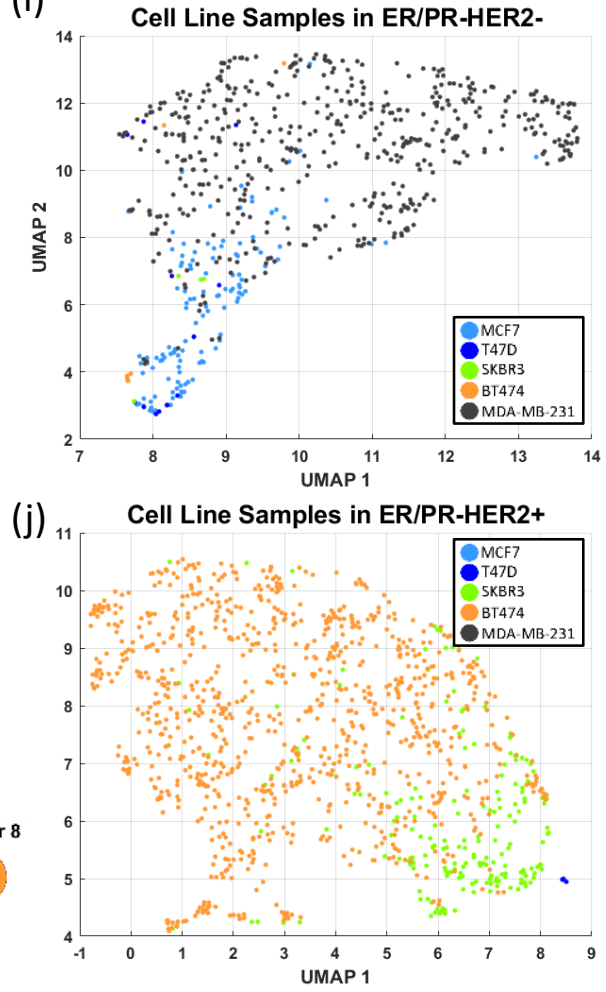

(k) ${ }_{9}$ Cell Line Samples in ER/PR+HER2+

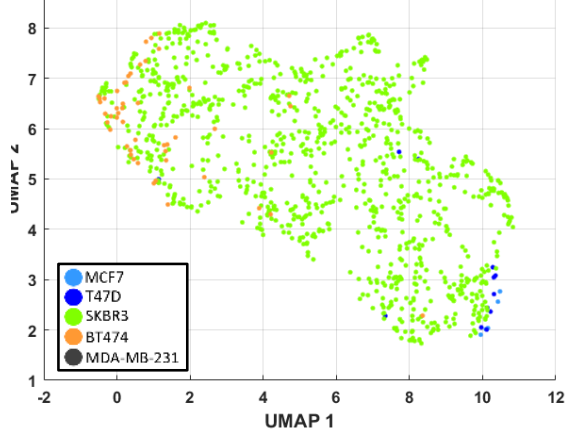

(I)

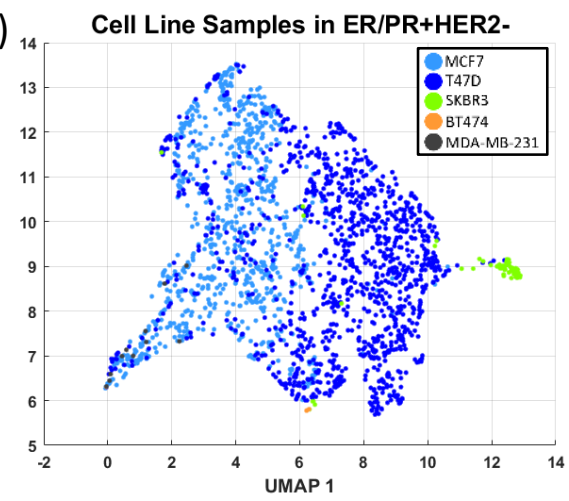

Figure 5. Characteristics of the identified subclusters of breast cancer cells. (a-d) Differences of the mean intensities of the subclusters in each breast cancer cell type. (e-h) UMAP visualization of hologram features color-coded with the subclusters. The pie plots indicate the proportion of the cell lines in each subcluster (the color code of cell lines are in (i-1)). (i-l) UMAP visualization of hologram features colorcoded with the cell lines. $*$ indicates the statistical significance $(\mathrm{p}<0.001)$ 
(a)

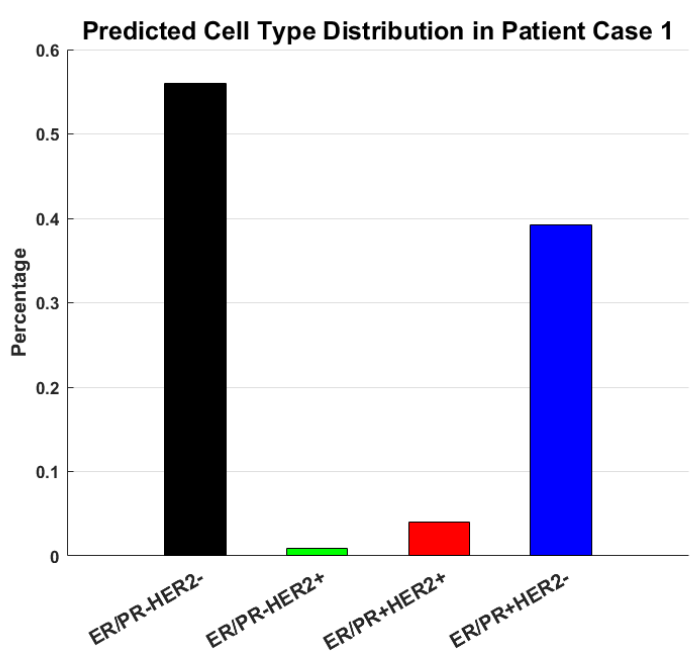

\begin{tabular}{|c|c|c|}
\hline Patient Case 1 & ER/PR- & ER/PR+ \\
\hline HER2- & $56 \%$ & $39.2 \%$ \\
\hline HER2+ & $0.8 \%$ & $4 \%$ \\
\hline \multicolumn{3}{|c}{$\mathbf{J}$} \\
\multicolumn{2}{|c}{}
\end{tabular}

ER/PR-HER2-

(b)

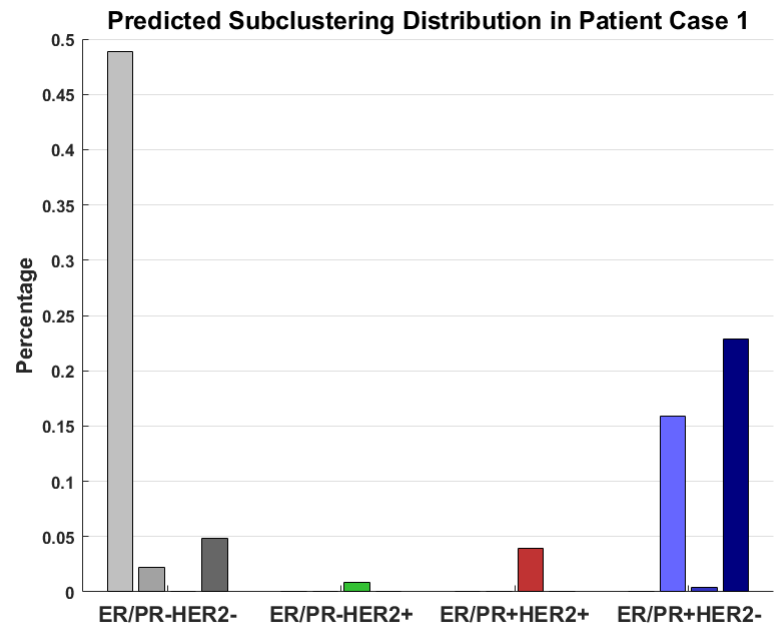

ER/PR-
HER2- $\left[\begin{array}{l}\text { Cluster 1 } \\ \text { Cluster 2 } \\ \text { Cluster 3 } \\ \text { Cluster 4 }\end{array} \quad\right.$ HER/PR- $\left[\begin{array}{r}\text { Cluster } 5 \\ \text { Cluster 6 } \\ \text { Cluster 7 } \\ \text { Cluster 8 }\end{array}\right.$

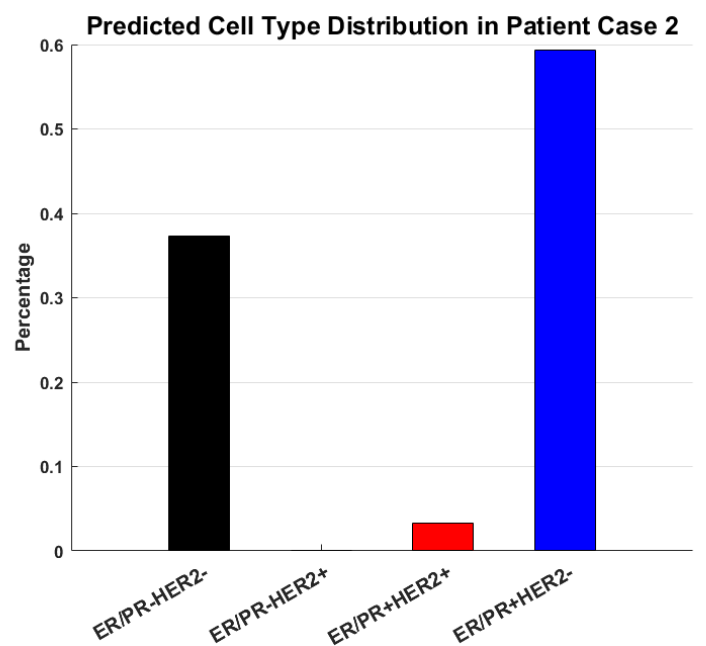

\begin{tabular}{|c|c|c|}
\hline Patient Case 2 & ER/PR- & ER/PR+ \\
\hline HER2- & $37.3 \%$ & $59.4 \%$ \\
\hline HER2+ & $0 \%$ & $3 \%$ \\
\hline \multicolumn{3}{|c}{$\mathbf{\downarrow}$} \\
\end{tabular}

ER/PR+HER2-

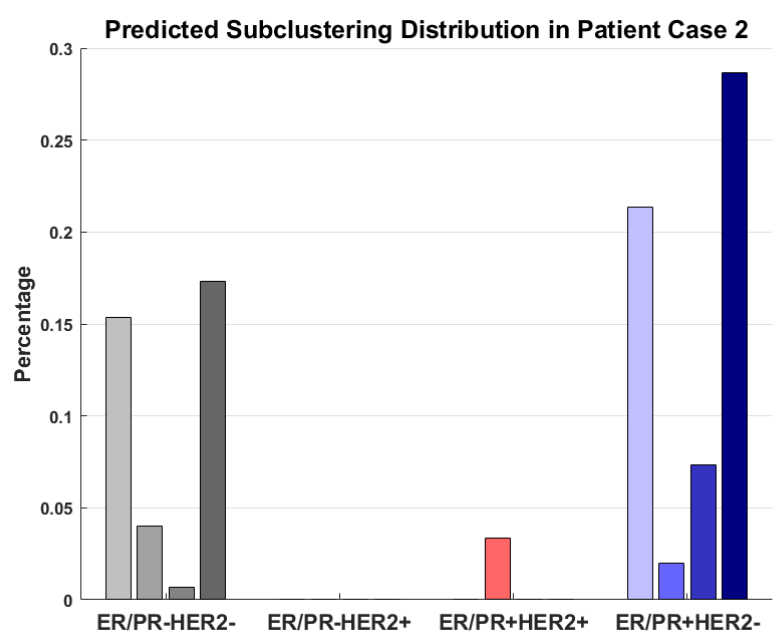

ER/PR+
HER2+ $\left[\begin{array}{cc}\text { Cluster } 9 \\ \text { Cluster 10 } 10 \\ \text { Cluster 11 }\end{array} \quad \begin{array}{r}\text { ER/PR+ } \\ \text { HER2- }\end{array}\left[\begin{array}{r}\text { Cluster } 12 \\ \text { Cluster 13 } \\ \text { Cluster 14 } \\ \text { Cluster 15 }\end{array}\right.\right.$

Figure 6. Profiling breast cancer cells from patient samples using the identified subclusters. (a) Distributions of the known types of breast cancer cells in two patients. (b) Distributions of the subclusters of breast cancer cells in two patients. 